\title{
On Certain Subclasses of Analytic Multivalent Functions Using Generalized Salagean Operator
}

\author{
Adnan Ghazy Alamoush and Maslina Darus \\ School of Mathematical Sciences, Faculty of Science and Technology, Universiti Kebangsaan Malaysia (UKM), \\ 43600 Bangi, Selangor, Malaysia
}

Correspondence should be addressed to Maslina Darus; maslina@ukm.edu.my

Received 16 February 2015; Revised 24 May 2015; Accepted 21 June 2015

Academic Editor: Patricia J. Y. Wong

Copyright ( 2015 A. G. Alamoush and M. Darus. This is an open access article distributed under the Creative Commons Attribution License, which permits unrestricted use, distribution, and reproduction in any medium, provided the original work is properly cited.

We introduce and study two subclasses of multivalent functions denoted by $\mathscr{M}_{p, n}^{m, \alpha, \beta, \sigma}\left(\lambda_{1} ; \lambda_{2}\right)$ and $\mathcal{N}_{p, n}^{m, \alpha, \beta, \sigma}(\mu, \delta ; \gamma)$. Further, by using the method of differential subordination, certain inclusion relations between the two subclasses aforementioned are given. Moreover, several consequences of the main results are also discussed.

\section{Introduction}

Let $\mathscr{A}_{(p, n)}$ denote the class of the functions $f$ of the form

$$
f(z)=z^{p}+\sum_{k=p+n}^{\infty} a_{k} z^{k}, \quad(n, p \in \mathbb{N}=\{1,2,3, \ldots\}),
$$

which are analytic in the open unit disc $\mathbb{U}=\{z \in \mathbb{C}:|z|<1\}$, and let denote $\mathscr{A}:=\mathscr{A}_{(1,1)}$.

A function $f \in \mathscr{A}_{(p, n)}$ is said to be multivalent starlike functions of order $\alpha$ in $\mathbb{U}$, if it satisfies the following inequality:

$$
\Re\left\{\frac{z f^{\prime}(z)}{f(z)}\right\}>\alpha, \quad z \in \mathbb{U},(0 \leq \alpha<p, p \in \mathbb{N}),
$$

and we denote this class by $S_{p, n}^{*}(\alpha)$.

A function $f \in \mathscr{A}_{(p, n)}$ is said to be multivalent convex functions of order $\alpha$ in $\mathbb{U}$, if it satisfies the following inequality:

$$
\mathfrak{R}\left\{1+\frac{z f^{\prime \prime}(z)}{f^{\prime}(z)}\right\}>\alpha, \quad z \in \mathbb{U},(0 \leq \alpha<p, p \in \mathbb{N}),
$$

and we denote this class by $C_{p, n}(\alpha)$.
For a function $f \in \mathscr{A}_{(p, n)}$, Goyal et al. [1] introduced the following generalized Salagean differential operator:

$$
\begin{aligned}
& D_{\sigma}^{0} f(z)=f(z), \\
& D_{\sigma}^{1} f(z)=D_{\sigma} f(z)=(1-\sigma) f(z)+\frac{\sigma}{p} z f^{\prime}(z),
\end{aligned}
$$

$(\sigma \geq 0)$,

$$
D_{\sigma}^{m} f(z)=D_{\sigma}\left(D_{\sigma}^{m-1} f(z)\right), \quad(m \in \mathbb{N}) .
$$

If $f$ is given by (1), then from (5) and (6) we have

$$
D_{\sigma}^{m} f(z)=z^{p}+\sum_{k=p+n}^{\infty}\left[1+\left(\frac{k}{p}-1\right) \sigma\right]^{m} a_{k} z^{k} .
$$

Remark 1. For $\sigma=p=1$, the differential operator $D_{\sigma}^{m} f(z)$ reduces to Salagean differential operator $D^{m} f(z)$ [2].

Definition 2. Let $\mathscr{M}_{p, n}^{m, \alpha, \beta, \sigma}\left(\lambda_{1} ; \lambda_{2}\right)$ be the class of functions $f \in \mathscr{A}_{(p, n)}$ that satisfy the condition 


$$
\begin{aligned}
& \mathfrak{R}\left\{\left(1-\lambda_{1}\right) \frac{z\left(D_{\sigma}^{\alpha, \beta, m} f(z)\right)^{\prime}}{D_{\sigma}^{\alpha, \beta, m} f(z)}\right. \\
& \left.\quad+\lambda_{1}\left(1+\frac{z\left(D_{\sigma}^{\alpha, \beta, m} f(z)\right)^{\prime \prime}}{\left(D_{\sigma}^{\alpha, \beta, m} f(z)\right)^{\prime}}\right)\right\}>\lambda_{2}, \quad z \in \mathbb{U},
\end{aligned}
$$

where $\left(0 \leq \sigma, 0 \leq \alpha<\beta \leq 1, \lambda_{1} \in \mathbb{R}, 0 \leq \lambda_{2}<p, m, p \in\right.$ $\mathbb{N})$, and let $\mathcal{N}_{p, n}^{m, \alpha, \beta, \sigma}(\mu, \delta ; \gamma)$ be the class of functions $f \in$ $\mathscr{A}_{(p, n)}$ that satisfy the conditions

$$
\begin{array}{r}
\frac{\left(D_{\sigma}^{\alpha, \beta, m} f(z)\right)\left(D_{\sigma}^{\alpha, \beta, m} f(z)\right)^{\prime}}{z^{2 p-1}} \neq 0, \quad z \in \mathbb{U}, \\
\mathfrak{R}\left\{\left(\frac{D_{\sigma}^{\alpha, \beta, m} f(z)}{z^{p}}\right)^{\mu}\left(\frac{\left(D_{\sigma}^{\alpha, \beta, m} f(z)\right)^{\prime}}{z^{p^{-1}}}\right)^{\delta}\right\}>\gamma, \quad z \in \mathbb{U},
\end{array}
$$

$$
\left(0 \leq \sigma, \delta, \mu \in \mathbb{R}, 0 \leq \alpha<\beta \leq 1,0 \leq \gamma<p^{\delta}(\alpha+\beta p)^{\delta+\mu}, m, p \in \mathbb{N}\right)
$$

where

$$
D_{\sigma}^{\alpha, \beta, m} f(z)=\alpha D_{\sigma}^{m} f(z)+\beta z\left(D_{\sigma}^{m} f(z)\right)^{\prime} .
$$

Remark 3. By specifying different values, we have some wellknown subclasses of the classes $A_{(p, n)}$ and $A_{(n)}=A_{(1, n)}$ appearing from the families of the classes $\mathscr{M}_{p, n}^{m, \alpha, \beta, \sigma}\left(\lambda_{1} ; \lambda_{2}\right)$ and $\mathcal{N}_{p, n}^{m, \alpha, \beta, \sigma}(\mu, \delta ; \gamma)$.

(i) $\mathscr{M}_{p, n}^{0,1,0, \sigma}\left(0 ; \lambda_{1}\right)=\mathcal{N}_{p, n}^{0,1,0, \sigma}\left(-1,1, \lambda_{1}\right)=S_{p, n}^{*},\left(0 \leq \lambda_{1}<\right.$ $p)$ is the class of multivalent starlike functions of order $\lambda_{1}$.

(ii) $\mathscr{M}_{1, n}^{0,1,0, \sigma}\left(0 ; \lambda_{1}\right)=\mathscr{N}_{1, n}^{0,1,0, \sigma}\left(-1,1, \lambda_{1}\right)=S_{1, n}^{*}=S_{n}^{*}, \quad(0 \leq$ $\left.\lambda_{1}<1\right)$ is the class of starlike functions of order $\lambda_{1}$.

(iii) $\mathscr{M}_{p, n}^{1,0,1,1}\left(0 ; \lambda_{1}\right)=\mathscr{C}_{p, n}\left(\lambda_{1}\right),\left(0 \leq \lambda_{1}<p\right)$ is the class of multivalent convex functions of order $\lambda_{1}$.

(iv) $\mathscr{M}_{1, n}^{1,0,1,1}\left(0 ; \lambda_{1}\right)=\mathscr{C}_{1, n}\left(\lambda_{1}\right)=\mathscr{C}_{n}\left(\lambda_{1}\right),\left(0 \leq \lambda_{1}<1\right)$ is the class of convex functions of order $\gamma$.

(v) $\mathscr{N}_{1, n}^{1,1,0,1}\left(1, \delta ; \lambda_{1}\right)=\mathscr{B}_{n}\left(\delta ; \lambda_{1}\right),\left(\delta \geq-1,0 \leq \lambda_{1}<1\right)$ is the subclass of Bazilević functions.

Let $\mathscr{H}[a, n]$ be denoted by the class

$$
\begin{aligned}
\mathscr{H} & {[a, n] } \\
& =\left\{h \in \mathscr{H}(\mathbb{U}): h(z)=a+a_{n} z^{n}+\cdots, z \in \mathbb{U}\right\} .
\end{aligned}
$$

In this investigation, we focus on certain inequalities consisting of the following differential operator $\mathcal{J}_{p, n}^{m, \alpha, \beta, \sigma}(\mu, \delta)$ : $\mathscr{A}_{(p, n)} \rightarrow \mathscr{H}[(\mu+\delta), n+p]:$

$$
\begin{aligned}
\mathcal{F}_{p, n}^{m, \alpha, \beta, \sigma}(\mu, \delta) f(z)= & \mu \frac{z\left(D_{\sigma}^{\alpha, \beta, m} f(z)\right)^{\prime}}{D_{\sigma}^{\alpha, \beta, m} f(z)} \\
& +\delta\left(1+\frac{z\left(D_{\sigma}^{\alpha, \beta, m} f(z)\right)^{\prime \prime}}{\left(D_{\sigma}^{\alpha, \beta, m} f(z)\right)^{\prime}}\right)
\end{aligned}
$$

that generalizes the expression used in the definition of class $\mathscr{M}_{p, n}^{m, \alpha, \beta, \sigma}\left(\lambda_{1} ; \lambda_{2}\right)$ and we receive several properties of the expression

$$
\left(\frac{D_{\sigma}^{\alpha, \beta, m} f(z)}{z^{p}}\right)^{\mu}\left(\frac{\left(D_{\sigma}^{\alpha, \beta, m} f(z)\right)^{\prime}}{z^{p-1}}\right)^{\delta}, \quad(z \in \mathbb{U}),
$$

including relations between classes $\mathscr{M}_{p, n}^{m, \alpha, \beta, \sigma}\left(\lambda_{1} ; \lambda_{2}\right)$ and $\mathcal{N}_{p, n}^{m, \alpha, \beta, \sigma}(\mu, \delta ; \gamma)$.

In order to prove our main results, we will need the following lemmas due to Miller and Mocanu [3].

Lemma 4. Let $\Omega \subset \mathbb{C}$ and suppose that the function $\psi: \mathbb{C}^{2} \times$ $\mathbb{U} \rightarrow \mathbb{C}$ satisfies $\psi\left(M e^{i \theta}, K e^{i \theta} ; z\right) \notin \Omega$ for all $K \geq M n, \theta \in$ $\mathbb{R}$, and $z \in \mathbb{U}$. If $h(z)=a+h_{n} z^{n}+\cdots$ is analytic in $\mathbb{U}$ and $\psi\left(h(z), z h^{\prime}(z) ; z\right) \in \Omega$ for all $z \in \mathbb{U}$, then $|h(z)|<M, z \in \mathbb{U}$.

Lemma 5. Let $\Omega \subset \mathbb{C}$ and suppose that the function $\psi: \mathbb{C}^{2} \times$ $\mathbb{U} \rightarrow \mathbb{C}$ satisfies $\psi(i x, y ; z) \notin \Omega$ for all $x \in \mathbb{R}, y \leq-n(1+$ $\left.x^{2}\right) / 2$, and $z \in \mathbb{U}$. If $h(z)=a+h_{n} z^{n}+\cdots$ is analytic in $\mathbb{U}$ and $\psi\left(h(z), z h^{\prime}(z) ; z\right) \in \Omega$ for all $z \in \mathbb{U}$, then $\Re\{h(z)\}>0, z \in \mathbb{U}$.

\section{Main Results}

Following the same techniques and procedure given by Goswami et al. [4], we have the following results.

Theorem 6. Let $f(z) \in \mathscr{A}_{(p, n)}$ with $\left(D_{\sigma}^{\alpha, \beta, m} f(z)\right)\left(D_{\sigma}^{\alpha, \beta, m} f(z)\right)^{\prime} /$ $z^{2 p-1} \neq 0$ for all $z \in \mathbb{U}$, where $D_{\sigma}^{\alpha, \beta, m}$ is given by (10), and also let $\mu, \delta \in \mathbb{R}$. If

$$
\begin{aligned}
\Re & \left\{\mathscr{J}_{p, n}^{m, \alpha, \beta, \sigma}(\mu, \delta) f(z)\right\} \\
& <p(\delta+\mu)+\frac{n M}{M+p^{\delta}(\alpha+\beta p)^{\delta+\mu}}, \quad(z \in \mathbb{U}),
\end{aligned}
$$


where $p^{\delta}(\alpha+\beta p)^{\delta+\mu} \leq M$, then

$$
\begin{gathered}
\mid\left(\frac{D_{\sigma}^{\alpha, \beta, m} f(z)}{z^{p}}\right)^{\mu}\left(\frac{\left(D_{\sigma}^{\alpha, \beta, m} f(z)\right)^{\prime}}{z^{p-1}}\right)^{\delta} \\
-p^{\delta}(\alpha+\beta p)^{\delta+\mu} \mid<M, \quad(z \in \mathbb{U}),
\end{gathered}
$$

where the powers are the principal ones.

Proof. Let the function $h(z)$ be defined by

$$
\begin{aligned}
h(z)= & \left(\frac{D_{\sigma}^{\alpha, \beta, m} f(z)}{z^{p}}\right)^{\mu}\left(\frac{\left(D_{\sigma}^{\alpha, \beta, m} f(z)\right)^{\prime}}{z^{p-1}}\right)^{\delta} \\
& -p^{\delta}(\alpha+\beta p)^{\delta+\mu} .
\end{aligned}
$$

From the assumptions $f \quad \in \quad \mathscr{A}_{(p, n)}$ with
$\left(D_{\sigma}^{\alpha, \beta, m} f(z)\right)\left(D_{\sigma}^{\alpha, \beta, m} f(z)\right)^{\prime} / z^{2 p-1} \neq 0$ for all $z \in \mathbb{U}$, we have that $h \in \mathscr{H}[0, n]$. By a simple manipulation, we have

$$
\begin{aligned}
\mathscr{J}_{p, n}^{m, \alpha, \beta, \sigma}(\mu, \delta) f(z)= & p(\delta+\mu) \\
& +\frac{z h^{\prime}(z)}{h(z)+p^{\delta}(\alpha+\beta p)^{\delta+\mu}} .
\end{aligned}
$$

Now letting

$$
\begin{aligned}
& \psi(r, s, z)=p(\delta+\mu)+\frac{s}{r+p^{\delta}(\alpha+\beta p)^{\delta+\mu}}, \\
& \Omega=\{w \in \mathbb{C}: \mathfrak{R}(w)<p(\delta+\mu) \\
& \left.+\frac{n M}{M+p^{\delta}(\alpha+\beta p)^{\delta+\mu}}\right\},
\end{aligned}
$$

we have from (17) and (14) that

$$
\psi\left(h(z), z h^{\prime}(z) ; z\right)=\mathscr{J}_{p, n}^{m, \alpha, \beta, \sigma}(\mu, \delta) f(z) \in \Omega
$$

$\forall z \in \mathbb{U}$.

Further, for any $\theta \in \mathbb{R}, K \geq n M$, and $z \in \mathbb{U}$, since $M \geq$ $p^{\delta}(\alpha+\beta p)^{\delta+\mu}$, we also have

$$
\begin{aligned}
\mathfrak{R} & \left\{\psi\left(M e^{i \theta}, K e^{i \theta} ; z\right)\right\} \\
& =p(\delta+\mu)+K \Re\left(\frac{1}{M+e^{-i \theta} p^{\delta}(\alpha+\beta p)^{\delta+\mu}}\right) \\
& \geq p(\delta+\mu)+\frac{n M}{M+p^{\delta}(\alpha+\beta p)^{\delta+\mu}}, \quad(z \in \mathbb{U}),
\end{aligned}
$$

which shows that $\psi\left(M e^{i \theta}, K e^{i \theta} ; z\right) \notin \Omega$ for all $\theta \in \mathbb{R}, K \geq$ $n M$, and $z \in \mathbb{U}$. Therefore, according to Lemma 4, we obtain $|h(z)|<M(z \in \mathbb{U})$. Hence, (15) is proven.

Theorem 7. Let $f(z) \in \mathscr{A}_{(p, n)}$ with $\left(D_{\sigma}^{\alpha, \beta, m} f(z)\right)\left(D_{\sigma}^{\alpha, \beta, m} f(z)\right)^{\prime} /$ $z^{2 p-1} \neq 0$ for all $z \in \mathbb{U}$, where $D_{\sigma}^{\alpha, \beta, m}$ is given by (10), and also let $\mu, \delta \in \mathbb{R}$. If

$$
\mathfrak{R}\left\{\mathscr{g}_{p, n}^{m, \alpha, \beta, \sigma}(\mu, \delta) f(z)\right\}>k(\mu, \delta, \alpha, \beta ; \gamma),
$$

$(z \in \mathbb{U})$,

where $\gamma \in\left[0, p^{\delta}(\alpha+\beta p)^{\delta+\mu}\right)$ and

$$
k(\mu, \delta, \alpha, \beta ; \gamma)= \begin{cases}p(\delta+\mu)-\frac{n \gamma}{2\left[p^{\delta}(\alpha+\beta p)^{\delta+\mu}-\gamma\right]}, & \text { if } \gamma \in\left[0, \frac{p^{\delta}(\alpha+\beta p)^{\delta+\mu}}{2}\right] \\ p(\delta+\mu)-\frac{n\left[p^{\delta}(\alpha+\beta p)^{\delta+\mu}-\gamma\right]}{2 \gamma}, & \text { if } \gamma \in\left[\frac{p^{\delta}(\alpha+\beta p)^{\delta+\mu}}{2}, p^{\delta}(\alpha+\beta p)^{\delta+\mu}\right),\end{cases}
$$

then $f \in \mathcal{N}_{p, n}^{m, \sigma, \alpha, \beta}(\mu, \delta ; \gamma)$.

Proof. Suppose that

$$
\left.\cdot\left(\frac{\left(D_{\sigma}^{\alpha, \beta, m} f(z)\right)^{\prime}}{z^{p-1}}\right)^{\delta}-\gamma\right]
$$

$$
h(z)=\frac{1}{p^{\delta}(\alpha+\beta p)^{\delta+\mu}-\gamma}\left[\left(\frac{D_{\sigma}^{\alpha, \beta, m} f(z)}{z^{p}}\right)^{\mu}\right.
$$

Then, $h(z)=1+h_{n} z^{n}+\cdots$ is analytic in $\mathbb{U}$. It is easily seen from (23) that 


$$
\begin{aligned}
& \mathcal{F}_{p, n}^{m, \alpha, \beta, \sigma}(\mu, \delta) f(z) \\
& =p(\delta+\mu)+\frac{\left(p^{\delta}(\alpha+\beta p)^{\delta+\mu}-\gamma\right) z h^{\prime}(z)}{\left(p^{\delta}(\alpha+\beta p)^{\delta+\mu}-\gamma\right) h(z)+\gamma} .
\end{aligned}
$$

Further, since

$$
\begin{gathered}
\psi(r, s ; z)=p(\delta+\mu)+\frac{\left(p^{\delta}(\alpha+\beta p)^{\delta+\mu}-\gamma\right) s}{\left(p^{\delta}(\alpha+\beta p)^{\delta+\mu}-\gamma\right) r+\gamma}, \\
\Omega=\{w \in \mathbb{C}: \mathfrak{R}(w)>k(\mu, \delta, \alpha, \beta ; \gamma)\},
\end{gathered}
$$

it leads to

$$
\psi\left(h(z), z h^{\prime}(z) ; z\right)=\mathcal{F}_{p, n}^{m, \alpha, \beta, \sigma}(\mu, \delta) f(z) \in \Omega
$$

$\forall z \in \mathbb{U}$.

Also, for any $x \in \mathbb{R}, y \leq-n\left(1+x^{2}\right) / 2$ and $z \in \mathbb{U}$, we have

$$
\begin{aligned}
& \mathfrak{R}\{\psi(i x, y ; z)\}=p(\delta+\mu)+\frac{\gamma\left(p^{\delta}(\alpha+\beta p)^{\delta+\mu}-\gamma\right) y}{\left[p^{\delta}(\alpha+\beta p)^{\delta+\mu}-\gamma\right]^{2} x^{2}+\gamma^{2}} \\
& \leq p(\delta+\mu) \\
& \quad-\frac{n \gamma\left[p^{\delta}(\alpha+\beta p)^{\delta+\mu}-\gamma\right]}{2} \frac{1+x^{2}}{\left[p^{\delta}(\alpha+\beta p)^{\delta+\mu}-\gamma\right]^{2} x^{2}+\gamma^{2}} \\
& \equiv q(z) \leq k(\mu, \delta, \alpha, \beta ; \gamma) \\
& = \begin{cases}\lim _{x \rightarrow \infty} q(z), & \text { if } \gamma \in\left[0, \frac{p^{\delta}(\alpha+\beta p)^{\delta+\mu}}{2}\right] \\
q(0), & \text { if } \gamma \in\left[\frac{p^{\delta}(\alpha+\beta p)^{\delta+\mu}}{2}, p^{\delta}(\alpha+\beta p)^{\delta+\mu}\right) ;\end{cases}
\end{aligned}
$$

that is, $\psi(i x, y ; z) \notin \Omega$. Finally, by Lemma 5 , we obtain that $\operatorname{Re}(h(z))>0$. The proof of Theorem 7 is complete.

\section{Corollaries and Consequences}

We will discuss some interesting consequences of the main theorems that extend some previous results obtained in ([4, 5]).

Putting $\alpha=1, \beta=0$ in Theorems 6 and 7, we get the following corollaries.

Corollary 8. Let $f(z) \in \mathscr{A}_{(p, n)}$ with $\left(D_{\sigma}^{m} f(z)\right)\left(D_{\sigma}^{m} f(z)\right)^{\prime} /$ $z^{2 p-1} \neq 0$ for all $z \in \mathbb{U}$, where $D_{\sigma}^{m}$ is given by (7), and also let $\mu, \delta \in \mathbb{R}$. If

$$
\begin{aligned}
& \Re\left\{\mu \frac{z\left(D_{\sigma}^{m} f(z)\right)^{\prime}}{D_{\sigma}^{m} f(z)}+\delta\left(1+\frac{z\left(D_{\sigma}^{m} f(z)\right)^{\prime \prime}}{\left(D_{\sigma}^{m} f(z)\right)^{\prime}}\right)\right\} \\
& <p(\delta+\mu)+\frac{n M}{M+p^{\delta}}, \quad(z \in \mathbb{U}),
\end{aligned}
$$

where $p^{\delta} \leq M$, then

$$
\left|\left(\frac{D_{\sigma}^{m} f(z)}{z^{p}}\right)^{\mu}\left(\frac{\left(D_{\sigma}^{m} f(z)\right)^{\prime}}{z^{p-1}}\right)^{\delta}-p^{\delta}\right|<M,
$$

$(z \in \mathbb{U})$,

where the powers are the principal ones.

Corollary 9. Let $f(z) \in \mathscr{A}_{(p, n)}$ with $\left(D_{\sigma}^{m} f(z)\right)\left(D_{\sigma}^{m} f(z)\right)^{\prime} /$ $z^{2 p-1} \neq 0$ for all $z \in \mathbb{U}$, where $D_{\sigma}^{m}$ is given by (7), and also let $\mu, \delta \in \mathbb{R}$. If

$$
\begin{aligned}
& \Re\left\{\mu \frac{z\left(D_{\sigma}^{m} f(z)\right)^{\prime}}{D_{\sigma}^{m} f(z)}+\delta\left(1+\frac{z\left(D_{\sigma}^{m} f(z)\right)^{\prime \prime}}{\left(D_{\sigma}^{m} f(z)\right)^{\prime}}\right)\right\} \\
& >\varphi(\mu, \delta ; \gamma), \quad(z \in \mathbb{U}),
\end{aligned}
$$

where $\gamma \in\left[0, p^{\delta}\right)$ and

$$
\begin{aligned}
& \varphi(\mu, \delta ; \gamma)=k(\mu, \delta, 1,0 ; \gamma) \\
& = \begin{cases}p(\delta+\mu)-\frac{n \gamma}{2\left[p^{\delta}-\gamma\right]}, & \text { if } \gamma \in\left[0, \frac{p^{\delta}}{2}\right] \\
p(\delta+\mu)-\frac{n\left[p^{\delta}-\gamma\right]}{2 \gamma}, & \text { if } \gamma \in\left[\frac{p^{\delta}}{2}, p^{\delta}\right),\end{cases}
\end{aligned}
$$

then

$$
\mathfrak{R}\left\{\left(\frac{D_{\sigma}^{m} f(z)}{z^{p}}\right)^{\mu}\left(\frac{\left(D_{\sigma}^{m} f(z)\right)^{\prime}}{z^{p-1}}\right)^{\delta}\right\}>\gamma, \quad(z \in \mathbb{U}),
$$

where the powers are the principal ones.

Taking $\mu=1-\lambda_{1}$ and $\delta=\lambda_{1}$ in Corollaries 8 and 9, respectively, we obtain the following special cases.

Corollary 10. Let $f(z) \in \mathscr{A}_{(p, n)}$ with $\left(D_{\sigma}^{m} f(z)\right)\left(D_{\sigma}^{m} f(z)\right)^{\prime} /$ $z^{2 p-1} \neq 0$ for all $z \in \mathbb{U}$, where $D_{\sigma}^{m}$ is given by (7), and also let $\lambda_{1} \in \mathbb{R}$. If

$$
\begin{aligned}
& \Re\left\{\left(1-\lambda_{1}\right) \frac{z\left(D_{\sigma}^{m} f(z)\right)^{\prime}}{D_{\sigma}^{m} f(z)}\right. \\
& \left.+\lambda_{1}\left(1+\frac{z\left(D_{\sigma}^{m} f(z)\right)^{\prime \prime}}{\left(D_{\sigma}^{m} f(z)\right)^{\prime}}\right)\right\}<p+\frac{n M}{M+p^{\lambda_{1}}},
\end{aligned}
$$

$(z \in \mathbb{U})$,

where $p^{\lambda_{1}} \leq M$, then

$$
\left|\left(\frac{D_{\sigma}^{m} f(z)}{z^{p}}\right)^{1-\lambda_{1}}\left(\frac{\left(D_{\sigma}^{m} f(z)\right)^{\prime}}{z^{p-1}}\right)^{\lambda_{1}}-p^{\lambda_{1}}\right|<M,
$$

$(z \in \mathbb{U})$,

where the powers are the principal ones. 
Corollary 11. Let $f(z) \in \mathscr{A}_{(p, n)}$ with $\left(D_{\sigma}^{m} f(z)\right)\left(D_{\sigma}^{m} f(z)\right)^{\prime} /$ $z^{2 p-1} \neq 0$ for all $z \in \mathbb{U}$, where $D_{\sigma}^{m}$ is given by (7), and also let $\lambda_{1} \in \mathbb{R}$. If

$$
\begin{aligned}
& \Re\left\{\left(1-\lambda_{1}\right) \frac{z\left(D_{\sigma}^{m} f(z)\right)^{\prime}}{D_{\sigma}^{m} f(z)}\right. \\
& \left.\quad+\lambda_{1}\left(1+\frac{z\left(D_{\sigma}^{m} f(z)\right)^{\prime \prime}}{\left(D_{\sigma}^{m} f(z)\right)^{\prime}}\right)\right\}>\chi\left(\lambda_{1} ; \gamma\right),
\end{aligned}
$$

$(z \in \mathbb{U})$,

where $\gamma \in\left[0, p^{\lambda_{1}}\right)$ and

$$
\begin{aligned}
\chi\left(\lambda_{1} ; \gamma\right) & =k\left(1-\lambda_{1}, \lambda_{1}, 1,0 ; \gamma\right) \\
& = \begin{cases}p-\frac{n \gamma}{2\left[p^{\lambda_{1}}-\gamma\right]}, & \text { if } \gamma \in\left[0, \frac{p^{\lambda_{1}}}{2}\right] \\
p-\frac{n\left[p^{\lambda_{1}}-\gamma\right]}{2 \gamma}, & \text { if } \gamma \in\left[\frac{p \lambda_{1}}{2}, p^{\lambda_{1}}\right),\end{cases}
\end{aligned}
$$

then

$$
\mathfrak{R}\left\{\left(\frac{D_{\sigma}^{m} f(z)}{z^{p}}\right)^{1-\lambda_{1}}\left(\frac{\left(D_{\sigma}^{m} f(z)\right)^{\prime}}{z^{p-1}}\right)^{\lambda_{1}}\right\}>\gamma,
$$

$(z \in \mathbb{U})$,

where the powers are the principal ones.

Next, upon taking $\alpha=0, \beta=1$ in Theorems 6 and 7, we obtain the following results.

Corollary 12. Let $f(z) \in \mathscr{A}_{(p, n)}$ with $\left(D_{\sigma}^{m} f(z)\right)^{\prime}\left[\left(D_{\sigma}^{m} f(z)\right)^{\prime}+\right.$ $\left.z\left(D_{\sigma}^{m} f(z)\right)^{\prime \prime}\right] / z^{2(p-1)} \neq 0$ for all $z \in \mathbb{U}$, where $D_{\sigma}^{m}$ is given by (7), and also let $\mu, \delta \in \mathbb{R}$. If

$$
\mathfrak{R}\left\{\mathscr{F}_{p, n}^{m, 0,1, \sigma}(\mu, \delta) f(z)\right\}<p(\delta+\mu)+\frac{n M}{M+p^{2 \delta+\mu}},
$$

$(z \in \mathbb{U})$,

where $p^{2 \delta+\mu} \leq M$, then

$$
\begin{aligned}
& \mid\left(\frac{\left(D_{\sigma}^{m} f(z)\right)^{\prime}}{z^{p-1}}\right)^{\mu}\left(\frac{\left(D_{\sigma}^{m} f(z)\right)^{\prime}+z\left(D_{\sigma}^{m} f(z)\right)^{\prime \prime}}{z^{p-1}}\right)^{\delta} \\
& -p^{2 \delta+\mu} \mid<M, \quad(z \in \mathbb{U}),
\end{aligned}
$$

where the powers are the principal ones.

Corollary 13. Let $f(z) \in \mathscr{A}_{(p, n)}$ with $\left(D_{\sigma}^{m} f(z)\right)^{\prime}\left[\left(D_{\sigma}^{m} f(z)\right)^{\prime}+\right.$ $\left.z\left(D_{\sigma}^{m} f(z)\right)^{\prime \prime}\right] / z^{2(p-1)} \neq 0$ for all $z \in \mathbb{U}$, where $D_{\sigma}^{m}$ is given by (7), and also let $\mu, \delta \in \mathbb{R}$. If

$$
\mathfrak{R}\left\{\mathscr{F}_{p, n}^{m, 0,1, \sigma}(\mu, \delta) f(z)\right\}>\phi(\mu, \delta ; \gamma), \quad(z \in \mathbb{U}),
$$

where $\gamma \in\left[0, p^{2 \delta+\mu}\right)$ and

$$
\begin{aligned}
& \phi(\mu, \delta ; \gamma)=k(\mu, \delta, 0,1 ; \gamma) \\
& = \begin{cases}p(\delta+\mu)-\frac{n \gamma}{2\left[p^{2 \delta+\mu}-\gamma\right]}, & \text { if } \gamma \in\left[0, \frac{p^{2 \delta+\mu}}{2}\right] \\
p(\delta+\mu)-\frac{n\left[p^{2 \delta+\mu}-\gamma\right]}{2 \gamma}, & \text { if } \gamma \in\left[\frac{p^{2 \delta+\mu}}{2}, p^{2 \delta+\mu}\right),\end{cases}
\end{aligned}
$$

then

$$
\begin{aligned}
& \Re\left\{\left(\frac{\left(D_{\sigma}^{m} f(z)\right)^{\prime}}{z^{p-1}}\right)^{\mu}\right. \\
& \left.\cdot\left(\frac{\left(D_{\sigma}^{m} f(z)\right)^{\prime}+z\left(D_{\sigma}^{m} f(z)\right)^{\prime \prime}}{z^{p-1}}\right)^{\delta}\right\}>\gamma,
\end{aligned}
$$

$(z \in \mathbb{U})$

where the powers are the principal ones.

Taking $\mu=1-\lambda_{1}$ and $\delta=\lambda_{1}$ in Corollaries 12 and 13, respectively, we obtain the following special cases.

Corollary 14. Let $f(z) \in \mathscr{A}_{(p, n)}$ with $\left(D_{\sigma}^{m} f(z)\right)^{\prime}\left[\left(D_{\sigma}^{m} f(z)\right)^{\prime}+\right.$ $\left.z\left(D_{\sigma}^{m} f(z)\right)^{\prime \prime}\right] / z^{2(p-1)} \neq 0$ for all $z \in \mathbb{U}$, where $D_{\sigma}^{m}$ is given by (7), and also let $\lambda_{1} \in \mathbb{R}$. If

$$
\mathfrak{R}\left\{\mathscr{F}_{p, n}^{m, 0,1, \sigma}\left(1-\lambda_{1}, \lambda_{1}\right) f(z)\right\}<p+\frac{n M}{M+p^{\lambda_{1}+1}},
$$

$(z \in \mathbb{U})$

where $p^{\lambda_{1}+1} \leq M$, then

$$
\begin{aligned}
& \mid\left(\frac{\left(D_{\sigma}^{m} f(z)\right)^{\prime}}{z^{p^{-1}}}\right)^{1-\lambda_{1}} \\
& \cdot\left(\frac{\left(D_{\sigma}^{m} f(z)\right)^{\prime}+z\left(D_{\sigma}^{m} f(z)\right)^{\prime \prime}}{z^{p-1}}\right)^{\lambda_{1}}-p^{\lambda_{1}+1} \mid<M, \\
& (z \in \mathbb{U}),
\end{aligned}
$$

where the powers are the principal ones.

Corollary 15. Let $f(z) \in \mathscr{A}_{(p, n)}$ with $\left(D_{\sigma}^{m} f(z)\right)^{\prime}\left[\left(D_{\sigma}^{m} f(z)\right)^{\prime}+\right.$ $\left.z\left(D_{\sigma}^{m} f(z)\right)^{\prime \prime}\right] / z^{2(p-1)} \neq 0$ for all $z \in \mathbb{U}$, where $D_{\sigma}^{m}$ is given by (7), and also let $\lambda_{1} \in \mathbb{R}$. If

$$
\mathfrak{R}\left\{\mathscr{F}_{p, n}^{m, 0,1, \sigma}\left(1-\lambda_{1}, \lambda_{1}\right) f(z)\right\}>\psi\left(\lambda_{1} ; \gamma\right),
$$




$$
\begin{aligned}
& \psi\left(\lambda_{1} ; \gamma\right)=k\left(1-\lambda_{1}, \lambda_{1}, 0,1 ; \gamma\right) \\
& = \begin{cases}p(\delta+\mu)-\frac{n \gamma}{2\left[p^{\lambda_{1}+1}-\gamma\right]}, & \text { if } \gamma \in\left[0, \frac{p^{\lambda_{1}+1}}{2}\right] \\
p(\delta+\mu)-\frac{n\left[p^{2 \delta+\mu}-\gamma\right]}{2 \gamma}, & \text { if } \gamma \in\left[\frac{p^{\lambda_{1}+1}}{2}, p^{\lambda_{1}+1}\right),\end{cases}
\end{aligned}
$$

then

$$
\begin{gathered}
\mathfrak{R}\left\{\left(\frac{\left(D_{\sigma}^{m} f(z)\right)^{\prime}}{z^{p^{-1}}}\right)^{1-\lambda_{1}}\right. \\
\left.\cdot\left(\frac{\left(D_{\sigma}^{m} f(z)\right)^{\prime}+z\left(D_{\sigma}^{m} f(z)\right)^{\prime \prime}}{z^{p-1}}\right)^{\lambda_{1}}\right\}>\gamma,
\end{gathered}
$$

$$
(z \in \mathbb{U})
$$

In the next result, we will find the relation between $\mathscr{M}_{p, n}^{m, \alpha, \beta, \sigma}\left(\lambda_{1} ; \gamma\right)$ and $\mathscr{M}_{p, n}^{m, \alpha, \beta, \sigma}\left(1-\lambda_{1}, \lambda_{1} ; \gamma\right)$. For this purpose, taking $\mu=1-\lambda_{1}$ and $\delta=\lambda_{1}$ in Theorem 7 , we obtain the following result.

Corollary 16. Let $f(z) \in \mathscr{A}_{(p, n)}$ with $\left(D_{\sigma}^{\alpha, \beta, m} f(z)\right)\left(D_{\sigma}^{\alpha, \beta, m} f(z)\right)^{\prime} /$ $z^{2 p-1} \neq 0$ for all $z \in \mathbb{U}$, where $D_{\sigma}^{\alpha, \beta, m}$ is given by (10), and also let $\lambda_{1} \in \mathbb{R}$. If

$$
f(z) \in \mathscr{M}_{p, n}^{m, \alpha, \beta, \sigma}\left(\lambda_{1} ; \varrho\left(\lambda_{1}, \alpha, \beta ; \gamma\right)\right)
$$

where $\gamma \in\left[0, p^{\lambda_{1}}(\alpha+\beta p)\right)$ and

where the powers are the principal ones.

$$
\varrho\left(\lambda_{1}, \alpha, \beta ; \gamma\right)=k\left(1-\lambda_{1}, \lambda_{1}, \alpha, \beta ; \gamma\right)= \begin{cases}p-\frac{n \gamma}{2\left[p^{\lambda_{1}}(\alpha+\beta p)-\gamma\right]}, & \text { if } \gamma \in\left[0, \frac{p^{\lambda_{1}}(\alpha+\beta p)}{2}\right] \\ p-\frac{n\left[p^{\lambda_{1}}(\alpha+\beta p)-\gamma\right]}{2 \gamma}, & \text { if } \gamma \in\left[\frac{p^{\lambda_{1}}(\alpha+\beta p)}{2}, p^{\lambda_{1}}[(\alpha+\beta p)),\right.\end{cases}
$$

then $f(z) \in \mathcal{N}_{p, n}^{m, \alpha, \beta, \sigma}\left(1-\lambda_{1}, \lambda_{1} ; \gamma\right)$.

Taking $\lambda_{1}=0$ and $n=1$ in the above corollary, we get the next special result.

Corollary 17. Let $f(z) \in \mathscr{A}_{(p)}$ with $\left(D_{\sigma}^{\alpha, \beta, m} f(z)\right)\left(D_{\sigma}^{\alpha, \beta, m} f(z)\right)^{\prime} /$ $z^{2 p-1} \neq 0$ for all $z \in \mathbb{U}$, where $D_{\sigma}^{\alpha, \beta, m}$ is given by (10), and also let $\lambda_{1} \in \mathbb{R}$. If

$$
f(z) \in \mathscr{M}_{p}^{m, \alpha, \beta, \sigma}(\varrho(\alpha, \beta ; \gamma)),
$$

where $\gamma \in[0, \alpha+\beta p)$ and

$$
\begin{aligned}
& \varrho(\alpha, \beta ; \gamma)=k(1,0, \alpha, \beta ; \gamma) \\
& = \begin{cases}p-\frac{\gamma}{2[(\alpha+\beta p)-\gamma]}, & \text { if } \gamma \in\left[0, \frac{\alpha+\beta p}{2}\right] \\
p-\frac{[(\alpha+\beta p)-\gamma]}{2 \gamma}, & \text { if } \gamma \in\left[\frac{\alpha+\beta p}{2},(\alpha+\beta p)\right),\end{cases}
\end{aligned}
$$

then $f(z) \in \mathcal{N}_{p}^{m, \alpha, \beta, \sigma}(1,0 ; \gamma)$.

Again, for the special cases of $\mu$ and $\delta$, Theorems 6 and 7 reduce at once to some results obtained by $[4,5]$.

Remark 18. Taking $p=1$ and $m=0$ in (7) and $\alpha=1$ and $\beta=0$ in (10), we get a known result obtained by Irmak et al. [5].

Remark 19. Taking $m=0$ in (7) and $\alpha=1-\beta$ in (10), we get a known result obtained by Goswami et al. [4].

\section{Conflict of Interests}

The authors declare that they have no competing interests.

\section{Authors' Contribution}

Both authors agreed with the contents of the paper.

\section{Acknowledgments}

The authors would like to acknowledge and appreciate the financial support received from Universiti Kebangsaan Malaysia (UKM) under Grant ERGS/1/2013/STG06/UKM/ $01 / 2$. The first author is also supported by ZAMALAH scheme grant under postgraduate center, UKM.

\section{References}

[1] S. P. Goyal, O. Singh, and P. Goswami, "Some relations between certain classes of analytic multivalent functions involving generalized salagean operator," Sohag Journal of Mathematics, vol. 1, no. 1, pp. 27-32, 2014.

[2] G. S. Salagean, "Subclasses of univalent functions," in Complex Analysis-Fifth Romanian-Finnish Seminar, Part 1: Proceedings of the Seminar Held in Bucharest 1981, vol. 1013 of Lecture Notes in Mathematics, pp. 362-372, Springer, Berlin, Germany, 1983.

[3] S. S. Miller and P. T. Mocanu, Differential Subordinations. Theory and Applications, , vol. 225 of Monographs and Textbooks in Pure and Applied Mathematics, Marcel Dekker, New York, NY, USA, 2000. 
[4] P. Goswami, T. Bulboacă, and S. Bansal, "Some relations between certain classes of analytic functions," Journal of Classical Analysis, vol. 1, no. 2, pp. 157-173, 2012.

[5] H. Irmak, T. Bulboacă, and N. Tuneski, "Some relations between certain classes consisting of $\alpha$-convex type and Bazilević type functions," Applied Mathematics Letters, vol. 24, no. 12, pp. 20102014, 2011. 


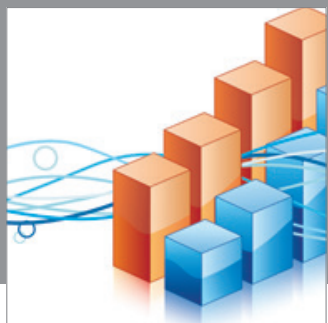

Advances in

Operations Research

mansans

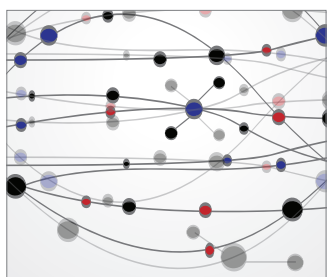

The Scientific World Journal
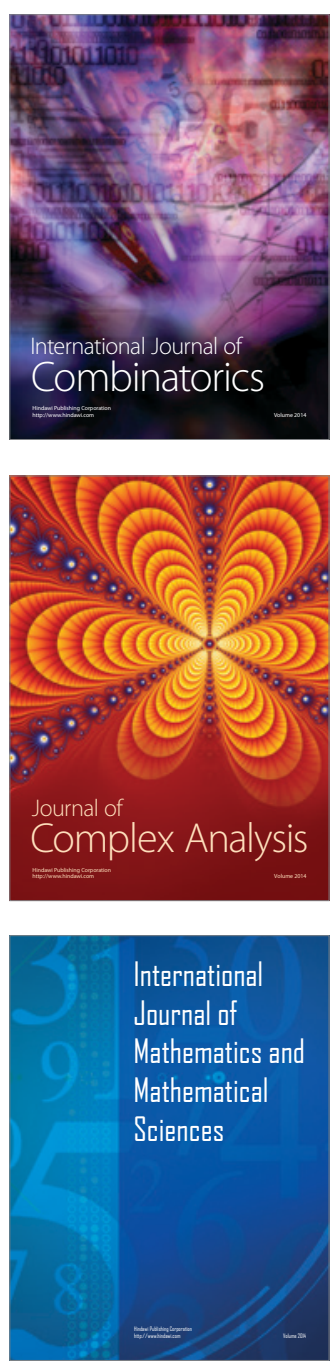
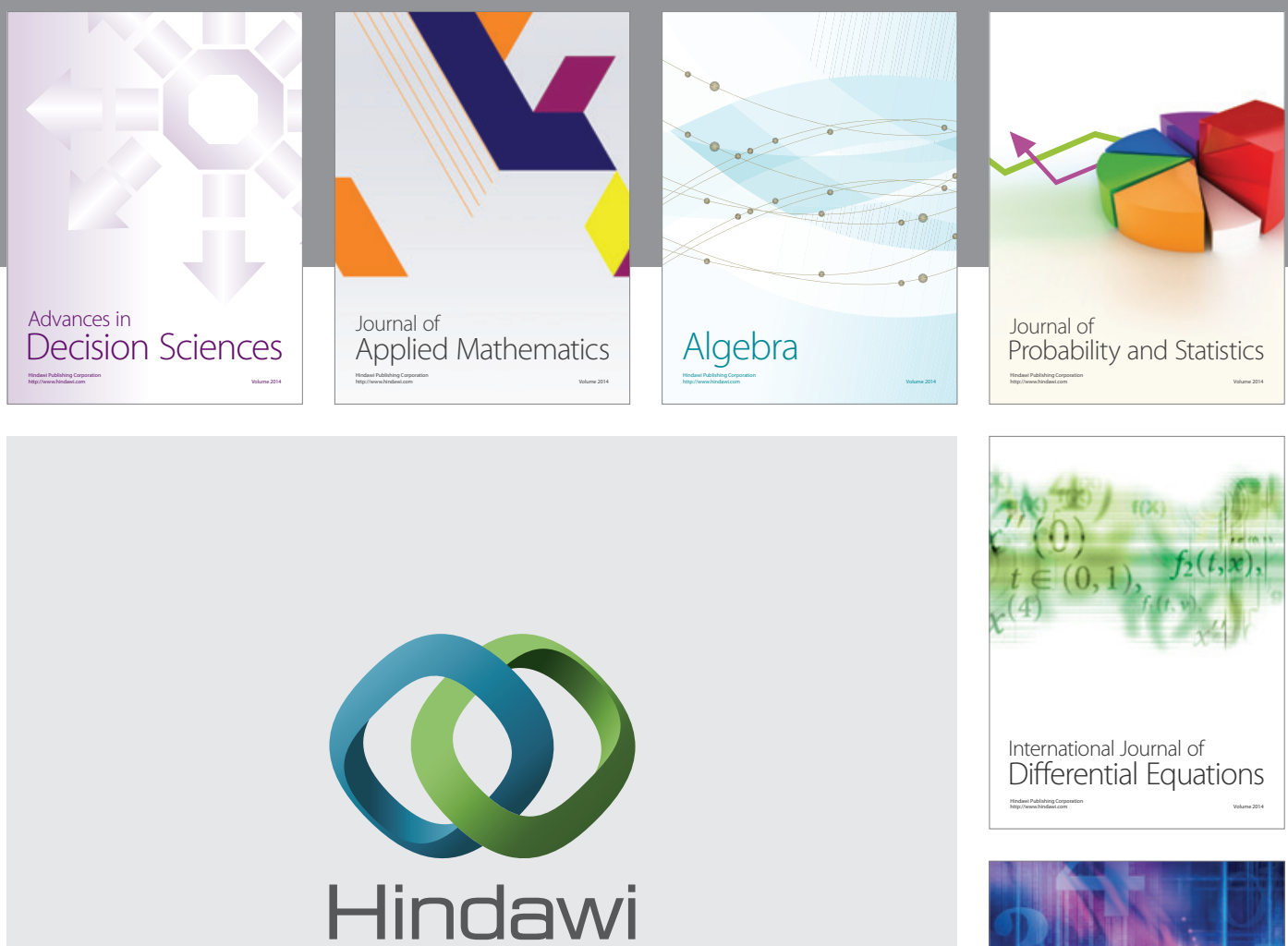

Submit your manuscripts at http://www.hindawi.com
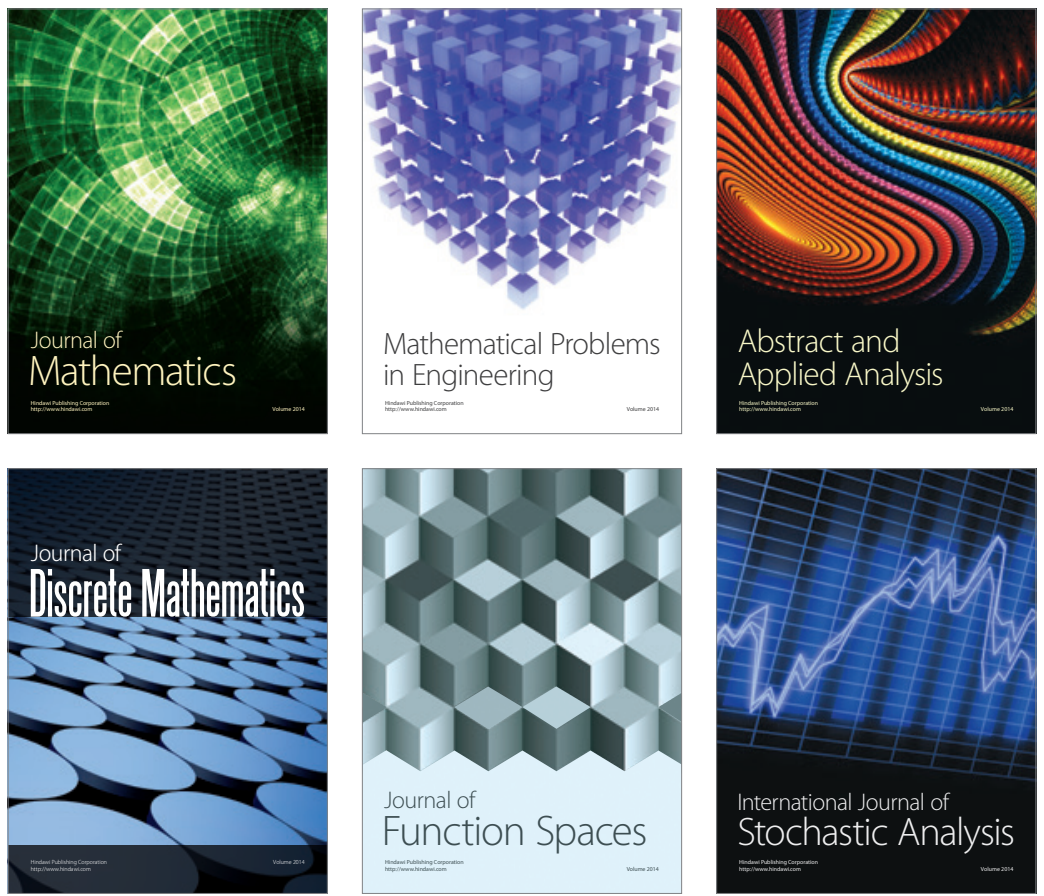

Journal of

Function Spaces

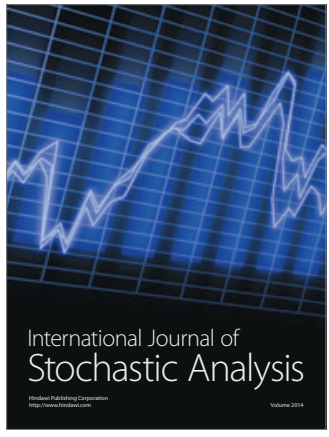

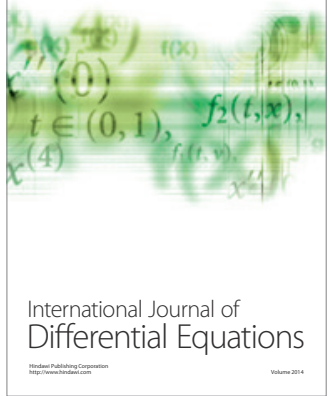
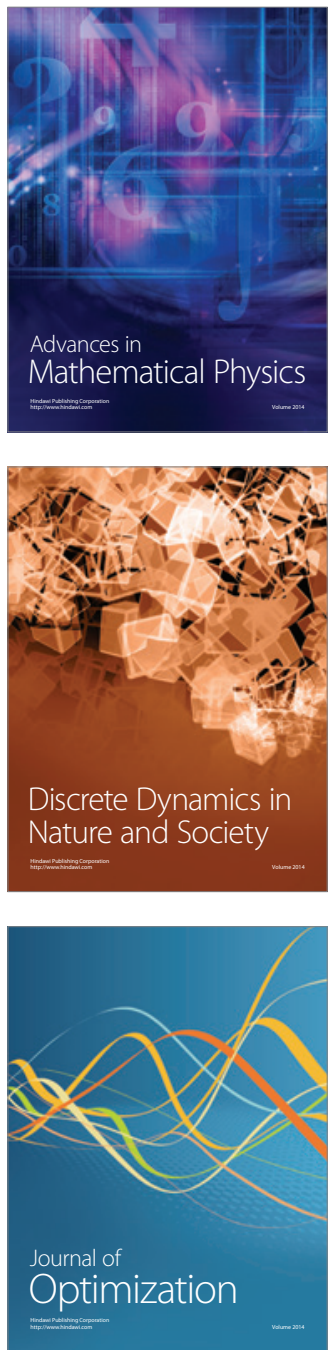